\title{
Reproductive potential and its behavioural consequences in orphaned bumblebee workers (Bombus impatiens)
}

\author{
Emily D. SibBald, Catherine M. S. Plowright \\ Ecole de psychologie, Université d'Ottawa, Ottawa, Ontario K1N 6N5, Canada \\ Received 14 October 2014 - Revised 3 January 2015 - Accepted 20 January 2015
}

\begin{abstract}
The supposition that aggression in orphaned workers is used in a battle over reproductive rights was evaluated for Bombus impatiens. Ovarian development was experimentally stimulated or inhibited in orphaned sisters. The manipulation translated into differences in egg laying. Two groups of pairs differed as to whether both or just one of the workers had developed ovaries. The prediction that workers with higher reproductive potential in the unmatched groups would show less aggression overall, compared to those in the matched groups, was not borne out. Nonetheless, butting frequency by the worker with developed ovaries was significantly reduced, as was oophagy, whereas the butting frequency by the worker with undeveloped ovaries was significantly increased. In contrast with Bombus terrestris, overt conflict does not end with one worker gaining a reproductive edge. The results challenge the premise that aggression is a means of preventing reproduction by kin: aggression may possibly serve to promote it.
\end{abstract}

\section{Bombus / reproduction / aggression / ovarian development / kin selection}

\section{INTRODUCTION}

Bumblebee workers, and social insects in general, are renowned for their altruism in sacrificing their own reproduction in favour of rearing sisters. There is conflict amongst sisters, however, and that conflict is revealed under circumstances that include the loss of control over their behaviour by the queen, either through the queen's advancing age, illness or death. Worker aggression (Bombus terrestris (L)., van der Blom 1986; Bloch 1999; Bombus bifarius Cresson, Foster et al. 2004) typically occurs within the context of worker reproduction (van Honk et al. 1981; Foster et al. 2004). Though workers are not mated, they lay unfertilized eggs, which, when reared, become males. Therein lies the potential for conflict: workers are more highly related to their own sons

Corresponding author: C. Plowright, cplowrit@uottawa.ca

Manuscript editor: Stan Schneider (coefficient of relatedness $=0.5$ ) than to their nephews (relatedness: 0.375 ) and so value their own reproduction more than that of their sisters. This is a specific instance of the conflict predicted by kin selection theory (Bourke and Ratnieks 2001; Foster et al. 2004; Ratnieks et al. 2006). Much current research is aimed at characterizing worker aggression and understanding its function, causes and consequences. For instance, 'worker policing' where one worker kills eggs laid by another, has recently been documented in several species of bees, wasps and ants (Ratnieks and Wenseleers 2005). Here, we examine conflict in queenless workers in a common North-American species of bumblebee, Bombus impatiens Cresson.

The hypothesis that aggression has a role in the competition for reproductive rights (Johnstone 2000) has been considered for several species of social insects (e.g. Bourke 1988a; Penick et al. 2013). In bumblebees, two lines of evidence support the hypothesis that aggression serves to monopolize reproduction. The first is the specificity of aggression: what is the target, where and when. 
In groups of orphaned $B$. impatiens workers, bees with developed ovaries are aggressive towards other workers, but fail to attack a honeybee intruder, leaving colony defence to others (Pomeroy 1981). Aggression is more likely in the absence of brood than in its presence, and the frequency of aggression by one worker is affected by the behavioural characteristics of the other (Sibbald and Plowright 2014). Conspecific males are also unlikely targets of aggression (Sibbald 2013). Aggression is more likely to occur around the time of oviposition (Sibbald and Plowright 2013), unless brood are present to elicit brood-directed behaviours.

The second line of evidence that aggression serves to assert reproductive rights is the link between ovarian development and aggression: more aggressive bees are, generally, more likely than less aggressive ones to have developed ovaries (Free 1955; Pomeroy 1981; Duchateau 1989; van Doorn 1989; Alaux et al. 2004) and to lay eggs (van Doorn and Heringa 1986; Alaux et al. 2004). The relationship between aggression and reproduction, however, is correlational and not necessarily causal. Both aggression and ovarian development may be the consequences of another proximal common cause: methylation in the genome (Amarasinghe et al. 2014).

Much of the research on worker conflict and reproduction has focused on the European species $B$. terrestris, and comparatively little is known about the North-American B. impatiens. Some comparisons of the behaviour of the two species across studies have been advanced, however, and have revealed some important differences. In intact colonies, the 'competition phase' between workers and the foundress queen is marked in B. terrestris (van Honk and Hogeweg 1981; Duchateau and Velthuis 1988) but there is little if any evidence for it in B. impatiens (Cnaani et al. 2002). There may be species differences in the relationship between space use by workers and ovarian development (Jandt and Dornhaus 2011). In our own observations of orphaned B. impatiens workers ( $1780 \mathrm{~h}$ of video and audio recordings in Sibbald and Plowright 2013), we have not detected the apparently threatening behaviours observed in B. terrestris, such as 'humming' (i.e. wing vibrations), that can occur before direct attacks (Duchateau 1989). In light of these species differences, we evaluated here the premise that aggression by one worker serves to curtail reproduction by her sister.

Here, we take an experimental approach to the study of orphaned sisters of B. impatiens. Pairs of workers isolated from the queen were used (as in Amsalem and Hefetz 2010; Sibbald and Plowright 2013, 2014). Whereas previous research treated ovarian development as a response variable (Cnaani et al. 2002; Sibbald and Plowright 2014), here, it was treated as an explanatory variable: we experimentally manipulated how well pairs were matched as to ovarian development. Egg laying was also monitored since ovarian development does not necessarily map onto reproductive behaviour (Duchateau and Velthuis 1989; van Doorn 1989; Foster et al. 2004). The prediction that we examined was that if one worker uses aggression to dissuade her sister from reproducing, then in an unmatched pair, where only one bee has developed ovaries and is at a reproductive advantage over her sister, there ought to be reduced conflict. In addition, the procedure allowed the disentanglement of two variables that are free to vary and confounded in nature: the reproductive status of one worker and that of her sister.

\section{METHODS}

\subsection{Colonies}

Three commercial B. impatiens colonies supplied by Biobest Canada Ltd. and one B. impatiens colony derived from a wild-caught queen and reared in the laboratory according to the procedures developed by Plowright and Jay (1966) were used between June and November 2010. Workers $(n=106)$ having hatched from their cocoon within the last $12 \mathrm{~h}$ (i.e. callow workers: bees with curved wings and without full colouration) were selected from the colonies and identified with coloured, numbered tags (Opalit Plättchen, The Bee Works, Orillia, ON) glued to the thorax. All workers and colonies were fed ad libitum pollen and honey-water solution ( $50 \%$ by volume), dispensed from feeder tubes that were monitored for signs of fermentation and changed daily. 


\subsection{Materials}

Once removed from their colonies, the workers were housed in wooden nesting boxes $(10.2 \times 10.2 \times 5.1 \mathrm{~cm})$ located in an incubator that maintained a temperature of $30{ }^{\circ} \mathrm{C}$. A fan circulated air above a basin of water to boost the humidity levels within the incubator and prevent desiccation within the nesting boxes. The nest box was lined with honeybee wax to produce a substrate for egg-cup building. It was connected to another box of the same dimensions containing a feeder tube. Both boxes were covered with glass plates. The laboratory was illuminated by a combination of natural and fluorescent light. Digital video recordings were made on a Vivotek IP8161 fixed network camera positioned directly over the nest box.

\subsection{Stimulation and inhibition of ovarian development}

To stimulate ovarian development in workers (heretofore referred to as $\mathrm{OD}+$ ), a modified protocol derived from Alaux et al. (2007) was used. The tagged callow workers were returned to their natal colonies for $48 \mathrm{~h}$ to allow for maturation. After which, they were removed from the colony and placed with a callow sister worker. The tagged worker was placed with the younger worker as older workers are more likely to be dominant egg layers in queenless groups (B. terrestris, Alaux et al. 2007). The two workers remained together in a nesting box in the incubator for 9 days to allow for ovarian development as it takes an average of 9 days for orphaned B. impatiens pairs to lay eggs (Sibbald 2013).

To inhibit ovarian development in workers (heretofore referred to as $\mathrm{OD}^{-}$), relative to the other group, after the $48 \mathrm{~h}$ of maturation in their natal colony, the tagged workers remained in the colony for an additional 9 days. Workers placed in intact colonies have reduced ovarian development compared to queenless groups (B. terrestris, Duchateau and Velthuis 1989).

As a manipulation check, 12 randomly selected OD+ workers and their partners were then dissected within $24 \mathrm{~h}$, as were $12 \mathrm{OD}^{-}$workers. The ovaries were weighed on a Mettler-Toledo balance (PB 303).

\subsection{Design}

After the period of ovarian stimulation or inhibition, the bees were paired in a new nest box under one of the following two conditions: (1) An OD+ worker paired with another $\mathrm{OD}+$ worker and (2) an $\mathrm{OD}+$ worker paired with an OD- worker. There were 10 pairs in each condition. All workers in each pair were of the same age (both hatched within $12 \mathrm{~h}$ of each other) and were from the same colony.

Oviposition was identified using the definition of Bloch and Hefetz (1999a): ' . . a worker seen inserting her abdomen into an open egg cup for a few minutes while moving it up and down, along with typical kicking movement of the hind legs. Oviposition behaviour was often preceded by a period of new egg cup construction or by opening and preparing an already existing one' (p. 127). Inter-individual aggression consists of butting and grappling behaviours, video examples of which are given by Sibbald and Plowright (2014) in the online supplementary materials. Butting is an accelerated movement of one bee towards the other resulting in contact and grappling is a joint somersault motion of two individuals (for further detail see Duchateau 1989; Sibbald and Plowright 2013, 2014). The initiator of each behaviour was identified and the duration of each act was measured. Significant inter-observer correlation between coders using these definitions has been obtained (Sibbald and Plowright 2013). We took note of oophagy, which is defined as the destruction of egg-cups and consumption of eggs (Owen and Plowright 1982; Ratnieks 1988; Cnaani et al. 2002; Alaux, et al. 2004). Ovaries were dissected and weighed within $24 \mathrm{~h}$ of the final day of testing.

\subsection{Procedure}

The recordings began immediately after an $\mathrm{OD}^{+}$ worker was paired with another OD+ or an OD- worker. Recordings were randomly started in the morning (anytime between 0730 hours-1030 hours), afternoon (1230 hours-1530 hours) and evening (1730 hours2030 hours) and continued for approximately $7 \mathrm{~h}$ for 7 days ( 977 h of video coded). Four coders, who were blind to the experimental conditions, undertook the behavioural coding.

\subsection{Data analysis}

The two groups of 10 pairs (OD+ with OD+; OD+ with $\mathrm{OD}^{-}$) generated the following comparisons. (1) To examine the effect of an individual's ovarian development on her own behaviour the $20 \mathrm{OD}+$ workers that 
were paired with each other (10 pairs) were compared to 10 OD- workers. The ovarian development of the partners in a pair is heretofore given in subscripts (so $\mathrm{OD}^{+}{ }_{(\mathrm{OD}+)}$ vs. $\left.\mathrm{OD}^{-}{ }_{(\mathrm{OD}+)}\right)$. (2) To examine the effect on an individual's behaviour of the individual's partner, the 20 OD+ workers that were paired with each other (10 pairs) were compared to the $10 \mathrm{OD}+$ workers that were

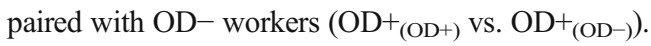

Masses are normally distributed and so comparisons were made using an analysis of variance and independent measures $t$ tests. Instances of inter-individual aggression (grapples and butts) are non-normally distributed rare events, and so a log-linear model, which specifies a Poisson error distribution, was fit to the data (McCullagh and Nelder 1983). The durations of grappling behaviour, which were positively skewed, were compared using Mood's median test. Because the frequency of oophagy is contingent upon the frequency of oviposition, we expressed instances of oophagy by one worker as a binomial proportion of ovipositions by the other (e.g. one worker showed four ovipositions, two of which were cannibalized). A logistic model, which specifies a binomial error term, was fit to the frequencies of oophagies.

The analyses were performed using SPSS Statistics 21. In keeping with Perneger (1998), Moran (2003) and Nakagawa (2004), we did not adopt the Bonferroni correction. $P$ values are presented as is.

\section{RESULTS}

\subsection{Manipulation check}

The results reported below (section 3.2) were not attributable to pre-experimental group differences in body mass. The mean body masses for the $12 \mathrm{OD}+$ workers $(\overline{\mathrm{x}}=0.228 \mathrm{~g}, \mathrm{SEM}=0.018)$ and the $12 \mathrm{OD}^{-}$workers $(\overline{\mathrm{x}}=0.189 \mathrm{~g}$, $\mathrm{SEM}=0.015$ ) that were dissected prior to the experiment were not significantly different $\left(t_{22}=1.70, P=0.10\right)$.

Figure 1 shows ovarian masses, expressed as a proportion of body mass, for the groups of workers $(n=12)$ that were dissected immediately after the manipulation of ovarian development. Group differences were significant $(F(2,35)=$ 49.62, $P<0.0001)$. Post hoc comparisons using a Tukey's HSD test revealed the OD- workers had significantly smaller (at least $5 \times$ smaller) ovaries
$(P<0.0001)$ than both the OD+ workers and the workers that had been placed with them when callow, though there was no significant difference between the latter two groups $(P=0.11)$.

\subsection{Experimental conditions}

\subsubsection{Ovarian development and oviposition}

Figure 2 shows ovarian masses, and Figure 3 shows the oviposition frequencies for the three groups of workers used in two comparisons. The two measures of reproduction paralleled each other. A comparison of the two groups of $\mathrm{OD}^{+}{ }_{(\mathrm{OD}+)}$ vs. $\mathrm{OD}^{+}{ }_{(\mathrm{OD}-)}$ showed that there was no significant effect of an OD+ worker's partner, either on ovarian mass $\left(t_{28}=0.60, P=0.56\right)$ or on rate of oviposition $\left(\chi^{2}=0.89, d f=1, P=0.35\right)$. The effect of ovarian development of the worker herself, however, was significant for both measures. The ovarian mass was significantly smaller for $\mathrm{OD}^{-}{ }_{(\mathrm{OD}+)}$ workers compared to $\mathrm{OD}^{+}{ }_{(\mathrm{OD}+)}\left(t_{28}=2.70\right.$, $P=0.012)$. The same was true for oviposition frequencies $\left(\chi^{2}=5.53, d f=1, P=0.019\right)$.

\subsubsection{Inter-individual aggression}

Figure 4 shows that the differences, described above, in ovarian mass and oviposition behaviour between the $\mathrm{OD}^{+}{ }_{(\mathrm{OD}+)}$ and $\mathrm{OD}^{-}{ }_{(\mathrm{OD}+)}$ workers did not translate into differences in overall interindividual aggression as measured by the total frequency of grapples and butts. The mean frequency of grapples and butts, combined, was comparable for the OD- and OD+ workers $\left(\chi^{2}=0.30, d f=1, P=0.58\right)$. No effect of the partner in a pair was detected $\left(\chi^{2}=3.34, d f=1, P=0.07\right)$.

The total aggression frequencies obscured a pattern: both measures of aggression did not 'behave' in the same way. In comparison with the $\mathrm{OD}_{(\mathrm{OD}+)}$ group, both other groups showed significantly different frequencies of butting. Having underdeveloped ovaries (OD-) had amplified $\left(\chi^{2}=7.99, d f=1, P=0.005\right)$ the frequency of butting. Having a partner with underdeveloped ovaries had the opposite effect: OD + workers were less likely to butt an OD- partner than an OD + partner $\left(\chi^{2}=10.68, d f=1, P=0.001\right)$. 


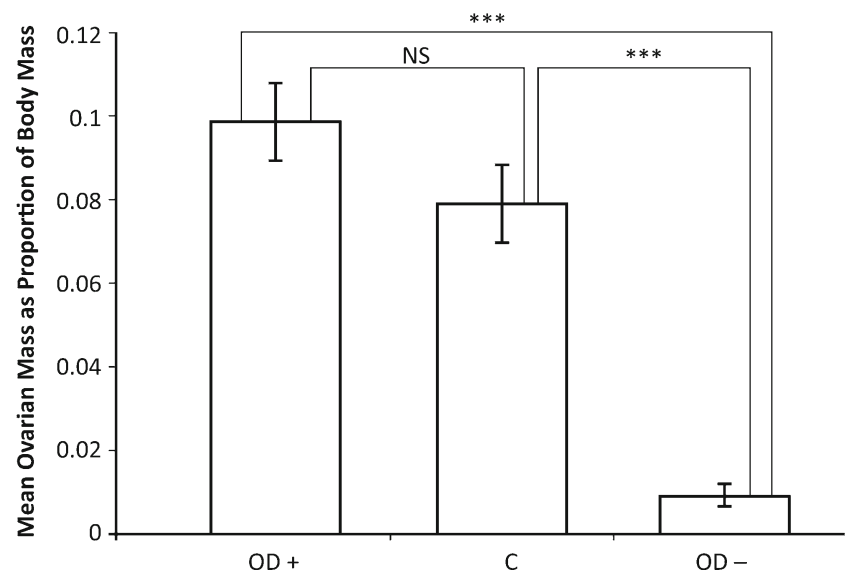

Figure 1. Mean ovarian mass, with standard error bars, expressed as a proportion of body mass, for three groups of bees ( $n=12$ per group) dissected to examine the effectiveness of the experimental manipulation of ovarian development. $O D+$ stimulated ovarian development, $C$ workers placed with the OD+ worker when callow, $O D$ - inhibited ovarian development. ${ }^{* * *} P<0.001$. NS no significant difference.

We considered the possibility that duration of aggressive acts would be a more sensitive measure than frequency. Mood's median test, however, revealed no significant difference with either of the other two groups $\left(\chi^{2} \leq 2.4, d f=1, P \geq 0.12\right)$.

\subsubsection{Oophagy}

The oviposition frequencies shown in Figure 3 are shown again in Figure 5. The frequencies of egg-laying bouts, however, are graphed not for the worker having laid the eggs, but for the other bee that had the opportunity to destroy and eat them. Ovarian development by one bee had no discernible effect on the tendency to destroy her sister's eggs: the proportions of ovipositions that were the object of oophagy were no different for $\mathrm{OD}_{(}{ }_{(\mathrm{OD}+)}$ vs. $\mathrm{OD}^{-}{ }_{(\mathrm{OD}+)}$ workers $\left(\chi^{2}=2.66, d f=1, P=0.10\right)$. A difference, however, emerged when compar-

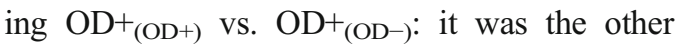
worker that had an effect on oophagy. When an

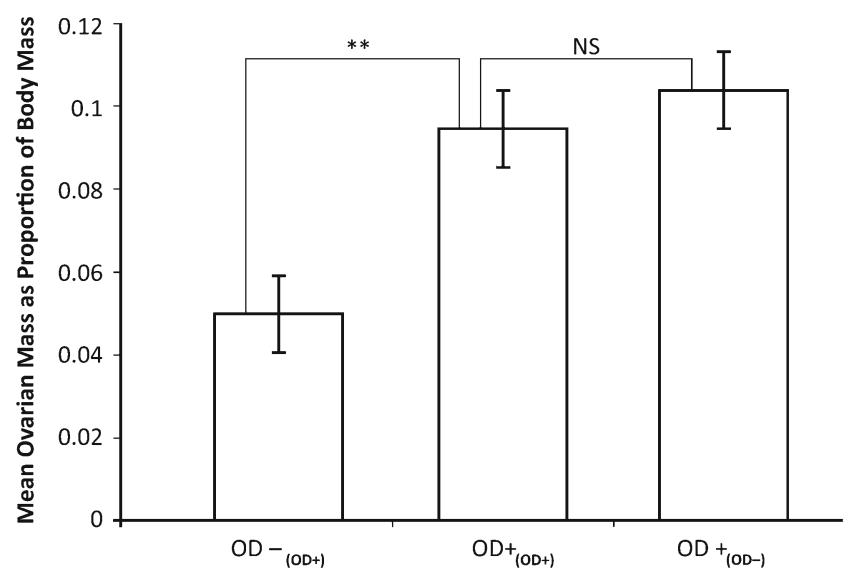

Figure 2. Mean ovarian mass, with standard error bars, expressed as a proportion of body mass. The horizontal axis shows whether the bee's ovarian development was stimulated $\left(O D^{+}\right)$or inhibited $\left(O D^{-}\right)$and the subscripts show whether the bee was paired with another $\mathrm{OD}+$ or $\mathrm{OD}-$ worker. The comparisons are based on data from two groups of 10 pairs of bees. ${ }^{* *}$ Significantly different $P<0.01$. NS not significant. 


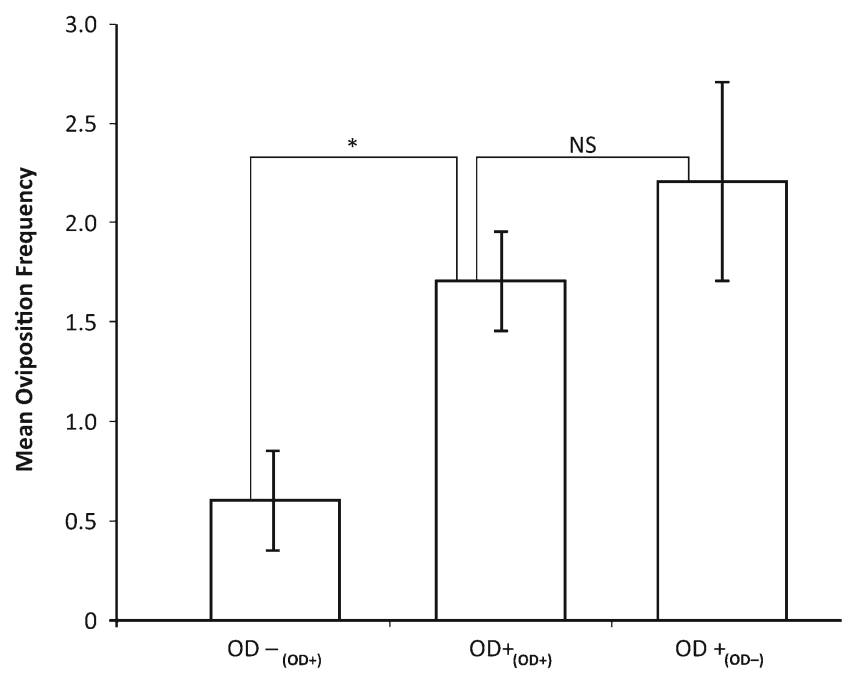

Figure 3. Mean frequency of oviposition (total number of ovipositions per bee), with standard error bars. The horizontal axis shows whether the bee's ovarian development was stimulated $\left(O D^{+}\right)$or inhibited $\left(O D^{-}\right)$and the subscripts show whether the bee was paired with another OD + or OD- worker. The comparisons are based on data from two groups of 10 pairs of bees. *Significantly different $P<0.05$. NS not significant.

OD+ worker was paired with another OD+ the proportion of instances of oophagy was 13 out of 34 ovipositions (38 \%). When an OD+ worker was paired with an OD- worker, however, that proportion fell to nil $\left(\chi^{2}=5.21, d f=1\right.$, $P=0.02)$ : out of six ovipositions, none were cannibalized. There is a possible scaling effect here. The finding that every single egg laid by the OD- workers was spared is based on a significantly smaller number of ovipositions by the OD- workers (section 3.2.1), only four of which had laid any eggs at all.

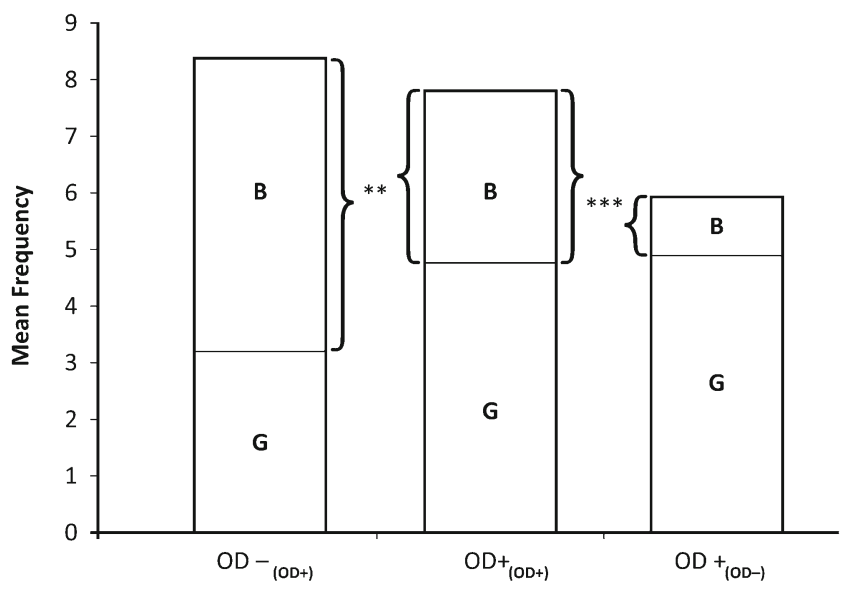

Figure 4. Mean frequency of inter-individual aggression (total number of incidences of aggression per bee): butting (B: top part of stacked bar) and grappling (G: bottom part of stacked bar ). The horizontal axis shows whether the bee's ovarian development was stimulated $(O D+)$ or inhibited $\left(O D^{-}\right)$and the subscripts show whether the bee was paired with another OD + or OD- worker. There were no significant differences in overall aggression, but there were significant differences in frequency of butting. The comparisons are based on data from two groups of 10 pairs of bees. $* * P<0.01, * * * P<0.001$. 


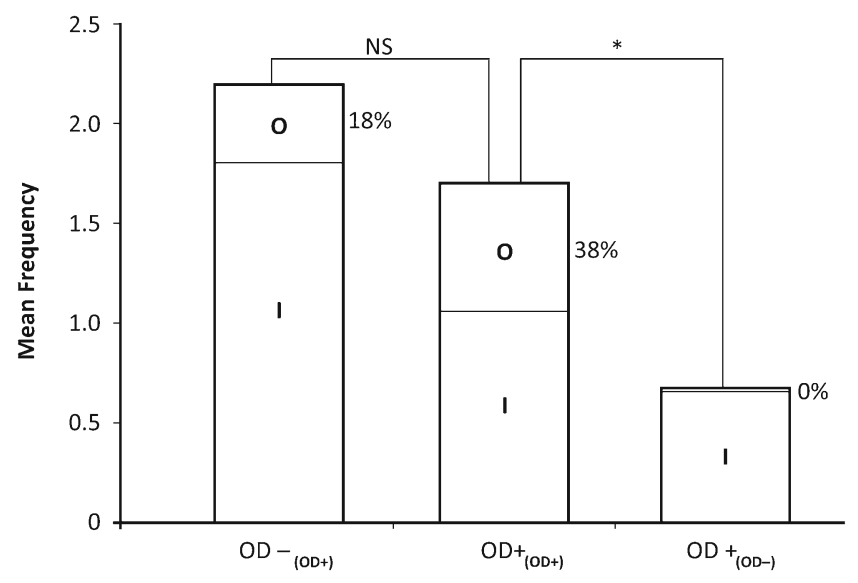

Figure 5. Mean frequency of oophagy by one worker (top part of stacked bar) as a proportion of mean frequency of ovipositions by the other worker (subscripted) in the pair. The total frequencies over the entire observation time were averaged across bees. $O$ ovipositions that were the object of oophagy, $I$ ovipositions left intact. The proportion $(\mathrm{O} /(\mathrm{O}+\mathrm{I}))$ is expressed as a percentage. The comparisons are based on data from two groups of 10 pairs of bees. *Significant difference in proportion $P<0.05$. NS no significant difference.

\section{DISCUSSION}

An orphaned worker that lays eggs is at a reproductive advantage over one that does not, providing of course that these eggs are themselves reared to generate individuals that can find mates and reproduce themselves. If inter-individual aggression were to serve the sole purpose of attaining this advantage, then it would seem counterproductive, in terms of time, energy and risk of injury, to persist in conflict once the reproductive advantage had been obtained. Though a previous study in this area on B. impatiens reported no conclusions regarding behavioural consequences of ovarian development because of infrequent agonistic encounters (Cnaani et al. 2007), our long sampling periods $(7 \mathrm{~h}$ /day instead of $0.5 \mathrm{~h}$ /day) enabled the capture of more of these rare events. Our results show that workers with stimulated ovarian development (OD+), though they laid more eggs than OD- workers, were not more likely to cease and desist in their aggressive interactions when they were in the company of an OD - worker than when they were paired with an OD+ worker. These results stand in contrast with those for some other eusocial insects. For example, once the main reproductive individual is established, behavioural enforcement can give way to chemical signalling (Cuvillier-Hot et al. 2002, 2004). In B. terrestris, there is some pheromonal control of reproduction (Bloch and Hefetz 1999b; Sramkova et al. 2008).

We propose two interpretations of our results on $B$. impatiens, both of which represent possible departures from what is known about worker conflict and reproduction in $B$. terrestris . One reading of our results is that two different behaviours, butting and grappling, may serve different functions. Though there were no differences in overall aggression, the frequency of butting was indeed significantly reduced for the $\mathrm{OD}+$ workers that were paired with OD- workers rather than with $\mathrm{OD}+$. This part of the results is in line with what is known about $B$. terrestris: egg-laying workers tend to be the recipients of aggression (van Doorn and Heringa 1986). Contrary to expectations, however, the OD- workers showed significantly higher, not lower, frequencies of butting. Given that they also destroyed and ate the eggs in almost one fifth of the ovipositions of the OD+ workers with which they were paired, perhaps, the grappling behaviours served to protect the eggs from eventual attack. Workers that lay fewer eggs, far from surrendering, as in B. terrestris (signalling 'I am out of the competition', Amsalem et al. 2009 , p. 1295), continue to compete for the 
opportunity to lay more, and workers that have achieved reproductive gains defend their eggs against attack. 'Dominance hierarchies' can be fluid and dynamic (van Doorn 1989).

Another interpretation is that the premise that the inter-individual aggression by one worker serves to prevent oviposition in another may be open to challenge. An individual's reproductive interests may be served by promoting rather than curtailing those of her sister. Although it is to a worker's advantage to produce sons rather than nephews (Bourke 1988b), if both workers can produce offspring at low cost to each other, it may be unnecessary to adopt behaviours that reduce the success of the other group members (Hammond and Keller 2004; Monnin and Ratnieks 1999). Indeed, using much the same methods as here, where food was unrestricted, we found a positive, not negative, correlation between the frequency of egg laying by one worker and that of another in pairs of orphaned sisters (Sibbald and Plowright 2013). This interpretation would also account for the finding that the few eggs laid by OD- workers were never destroyed. Perhaps from the perspective of view of their sisters, the OD- workers were not laying too many eggs, but rather were not laying enough. Given that the logic of kin selection is a key element of this line of reasoning, future investigations might incorporate manipulations of kin structure (see Takahashi et al. 2010), as well as costs and benefits of reproduction by one individual to the other.

This study involved long observation periods and time consuming coding of behavioural details. It harnessed the variable of reproductive status that naturally varies freely between individuals. This approach may not lend itself well to tracking entire colonies over their life cycle, though it is possible to examine the effects of increasing the group size of a few isolated workers (Cnaani et al. 2007 for B. impatiens; Amsalem and Hefetz 2011 for B. terrestris). With larger groups may come increased incentive to forage, which in turn may intensify reproductive competition. Our point here was not to argue that the behaviour in pairs of workers is necessarily representative of that of larger groups, but rather to suggest that aggression may not necessarily serve to curb reproduction. Moreover, an understanding of the relevant factors might be achieved by working up from the simplest situation, as well as working down from the most complex.

One caveat in the interpretation of our results is that the manipulation of reproductive status our study was achieved by the non-invasive and biological realistic means of differential exposure to the intact colony over 9 days prior to testing. The differences in social conditions that affected the change in ovarian development, however, may also have affected the workers in other ways. One possible criticism regards the extent to which the conditions in the lab may not reflect those in nature. Some of the honeybee wax on the floor of the nest box that was provided for traction may conceivably have been incorporated into egg-cups. The 24-h exposure to light was also artificial, even though performance of nest tasks is not as influenced by diurnal rhythms as performance of foraging tasks (Yerushalmi et al. 2006). Given that both members of each pair were observed on the same substrate under the same lighting conditions, however, these common aspects of the procedure are unlikely explanations for the root causes of the behavioural differences we reported.

Acceptance, tolerance or rejection by workers of the reproductive efforts of other workers almost certainly has consequences for colony development. A comparison across studies in colony growth between B. terrestris and B. impatiens, advanced by Cnaani et al. (2002), suggested that $B$. impatiens colonies may, on average, grow to have more workers but fewer reproductives. Nonetheless, given the variability in reproductive output across colonies (e.g. Weinberg 2007), and its dependence on rearing conditions (Duchateau and Velthuis 1988), such generalizations remain tentative.

In this paper, we have reported data on one commercially available species and suggested possible differences with another. These differences remain to be confirmed in side-by-side comparisons within experiments. There are, however, about 250 Bombus species worldwide (Williams and Osborne 2009), most of which remain to be drawn into the picture. 


\section{ACKNOWLEDGMENTS}

This manuscript was prepared as part of the first author's doctoral thesis. The work was funded by a research grant to C.M.S.P. from the Natural Sciences and Engineering Research Council of Canada and a graduate scholarship to E.S. from the Fonds de recherche sur la nature et les technologies du Québec. We thank Michael Richards for the construction of testing equipment and software development, Dwayne Schindler for the statistical advice, Daniel Dostie for the graphic design, Robert Laganière for his assistance with software engineering and Nelson Pomeroy and Chris Plowright for invaluable discussions on the behaviour of orphaned bumblebees. Robin Foster, Levente Orbán and Emma Thompson provided a constructive criticism on the manuscript. Joseph Nesrallah, Balázs Orbán, Emma Thompson and Robert Zadra assisted with data coding. Biobest Canada Ltd. kindly donated colonies for our research.

Le potentiel de reproduction et ses conséquences sur le comportement d'ouvrières orphelines chez les bourdons .

Bombus / reproduction / aggression / développement ovarien / sélection de parentèle

Das Reproduktionspotential und seine Konsequenzen auf das Verhalten weiselloser Arbeiterinnen der Hummel Bombus impatiens

Bombus / Reproduktion / Aggression / Ovarentwicklung / Verwandtenselektion

\section{REFERENCES}

Alaux, C., Jaisson, P., Hefetz, A. (2004a) Queen influence on worker reproduction in bumblebees (Bombus terrestris ) colonies. Insectes Soc. 51, 287-293

Alaux, C., Boutot, M., Jaisson, P., Hefetz, A. (2007) Reproductive plasticity in bumblebee workers (Bombus terrestris ) - reversion from fertility to sterility under queen influence. Behav. Ecol. Sociobiol. 62 , 213-222

Amarasinghe, H.E., Clayton, C.I., Mallon, E.B. (2014) Methylation and worker reproduction in the bumblebee (Bombus terrestris). Proc. R. Soc. Lond. B 281, 20132502

Amsalem, E., Hefetz, A. (2010) The appeasement effect of sterility signaling in dominance contests among Bombus terrestris workers. Behav. Ecol. Sociobiol. 64, 1685-1694
Amsalem, E., Hefetz, A. (2011) The effect of group size on the interplay between dominance and reproduction in Bombus terrestris. PLoS ONE 6(3), e18238

Amsalem, E., Twele, R., Francke, W., Hefetz, A. (2009) Reproductive competition in the bumble-bee Bombus terrestris : do workers advertise sterility? Proc. R. Soc. Lond. B 276, 1295-1304

Bloch, G. (1999) Regulation of queen-worker conflict in bumble-bee (Bombus terrestris) colonies. Proc. R. Soc. Lond. B 266, 2465-2469

Bloch, G., Hefetz, A. (1999a) Regulation of reproduction by dominant workers in bumblebee (Bombus terrestris) queenright colonies. Behav. Ecol. Sociobiol. 45, 125-135

Bloch, G., Hefetz, A. (1999b) Reevaluation of the role of mandibular glands in regulation of reproduction in bumblebee colonies. J. Chem. Ecol. 25, 881-896

Bourke, A.F.G. (1988a) Dominance orders, worker reproduction, and queen-worker conflict in the slavemaking ant Harpagoxenus sublaevis. Behav. Ecol. Sociobiol. 23, 323-333

Bourke, A.F.G. (1988b) Worker reproduction in the higher eusocial Hymenoptera. Q. Rev. Biol. 63, 291-311

Bourke, A.F.G., Ratnieks, F.L.W. (2001) Kin-selected conflict in the bumble-bee Bombus terrestris (Hymenoptera: Apidae). Proc. R. Soc. Lond. B 268 (1465), 347355

Cnaani, J.R., Schmid-Hempel, R., Schmidt, J.O. (2002) Colony development, larval development and worker reproduction in Bombus impatiens Cresson. Insectes Soc 49, 164-170

Cnaani, J., Wong, A., Thomson, J.D. (2007) Effect of group size on ovarian development in bumblebee workers (Hymenoptera: Apidae: Bombus). Entomol. Gen. 29, 305-314

Cuvillier-Hot, V., Gadagkar, R., Peeters, C., Cobb, M. (2002) Regulation of reproduction in a queenless ant: Aggression, pheromones and reduction in conflict. Proc. R. Soc. Lond. B 269, 1295-1300

Cuvillier-Hot, V., Lenoir, A., Crewe, R., Malosse, C., Peeters, C. (2004) Fertility signalling and reproductive skew in queenless ants. Anim. Behav. 68, 1209-1219

Duchateau, M.J. (1989) Agonistic behaviours in colonies of the bumblebee Bombus terrestris. J. Ethol. 7, 141-151

Duchateau, M.J., Velthuis, H.H.W. (1988) Development and reproductive strategies in Bombus terrestris colonies. Behaviour 107, 186-207

Duchateau, M.J., Velthuis, H.H.W. (1989) Ovarian development and egg laying in workers of Bombus terrestris . Entomol. Exp. App. 51 , 199-213

Foster, R.L., Brunskill, A., Verdirame, D., O’Donnell, S. (2004) Reproductive physiology, dominance interactions, and division of labour among bumble bee workers. Physiol. Entomol. 29, 327-334

Free, J.B. (1955) The behaviour of egg-laying workers of bumblebee colonies. Br. J. Anim. Behav. 3, 147-153 
Hammond, R.L., Keller, L. (2004) Conflict over male parentage in social insects. PLoS Biol., e248.

Jandt, J.M., Dornhaus, A. (2011) Competition and cooperation: bumblebee spatial organization and division of labor may affect worker reproduction late in life. Behav. Ecol. Sociobiol. 65, 2341-2349

Johnstone, R.A. (2000) Models of reproductive skew: A review and synthesis. Ethology 106, 5-26

McCullagh, P., Nelder, J.A. (1983) Generalized Linear Models. Chapman and Hall, London

Monnin, T., Ratnieks, F.L.W. (1999) Reproduction versus work in queenless ants: When to join a hierarchy of hopeful reproductives? Behav. Ecol. Sociobiol. 46, 413-422

Moran, M.D. (2003) Arguments for rejecting the sequential Bonferroni in ecological studies. Oikos 100, 403-405

Nakagawa, S. (2004) A farewell to Bonferroni: The problems of low statistical power and publication bias. Behav. Ecol. 15, 1044-1045

Owen, R.E., Plowright, R.C. (1982) Worker-queen conflict and male parentage in bumble bees. Behav. Ecol. Sociobiol. 11, 91-99

Penick, C.A., Trobaugh, B., Brent, C.S., Liebig, J. (2013) Head-butting as an early indicator of reproductive disinhibition in the termite Zootermopsis nevadensis. J. Insect Behav. 26, 23-34

Perneger, T.V. (1998) What's wrong with Bonferroni adjustments. Br. Med. J. 316, 1236-1238

Plowright, R.C., Jay, S.C. (1966) Rearing bumble bee colonies in captivity. J. Apic. Res. 5, 155-165

Pomeroy, N. (1981) Reproductive dominance interactions and colony development in bumble bees (Bombus Latreille; Hymenoptera: Apidae). (Doctoral dissertation, University of Toronto). Diss. Abstr. Int. B 42, 3981

Ratnieks, F.L. (1988) Reproductive harmony via mutual policing by workers in eusocial Hymenoptera. Am. Nat. 132, 217-236

Ratnieks, F.L., Wenseleers, T. (2005) Policing insect societies. Science 307 (5706), 54-56

Ratnieks, F.L., Foster, K., Wenseleers, T. (2006) Conflict resolution in insect societies. Annu. Rev. Entomol. 51, 581-608

Sibbald, E.D. (2013) Aggression, social Interactions, and reproduction in orphaned (Bombus impatiens) workers: Defining dominance. Doctoral dissertation, University of Ottawa.

Sibbald, E.D., Plowright, C.M.S. (2013) On the relationship between aggression and reproduction in pairs of orphaned worker bumblebees (Bombus impatiens). Insectes Soc. 60, 23-30

Sibbald, E.D., Plowright, C.M.S. (2014) Social interactions and their connection to aggression and ovarian development in orphaned worker bumblebees (Bombus impatiens). Behav. Process. 103, $150-155$

Sramkova, A., Schulz, C., Twele, R., Francke, W., Ayasse, M. (2008) Fertility signals in the bumblebee Bombus terrestris (Hymenoptera: Apidae). Naturwissenschaften 95, 515-522

Takahashi, J.I., Martin, S.J., Ono, M., Shimizu, I. (2010) Male production by non-natal workers in the bumblebee, Bombus deuteronymus (Hymenoptera: Apidae). J. Ethol. 28, 61-66

van der Blom, J. (1986) Reproductive dominance within colonies of Bombus terrestris (L.). Behaviour 97, 37-49

van Doorn, A. (1989) Factors influencing dominance behaviour in queenless bumblebee workers (Bombus terrestris ). Physiol. Entomol. 14, 211-221

van Doorn, A., Heringa, J. (1986) The ontogeny of a dominance hierarchy in colonies of the bumblebee Bombus terrestris (Hymenoptera, Apidae). Insectes Soc. 33, 3-25

van Honk, C.G.J., Hogeweg, P. (1981) The ontogeny of the social structure in a captive Bombus terrestris colony. Behav. Ecol. Sociobiol. 9, 111-119

van Honk, C.G.J., Röseler, P.F., Velthuis, H.H.W., Hoogeveen, J.C. (1981) Factors influencing the egg laying of workers in a captive Bombus terrestris colony. Behav. Ecol. Sociobiol. 9, 9-14

Weinberg, D. (2007) Simultaneous deprivation of pollen and nectar: Pollen collection behaviour and development in bumblebee colonies (Bombus impatiens Cresson). (Doctoral dissertation, University of Ottawa). -

Williams, P.H., Osborne, J.L. (2009) Bumblebee vulnerability and conservation world-wide. Apidologie 40, 367-387

Yerushalmi, S., Bodenhaimer, S., Bloch, G. (2006) Developmentally determined attenuation in circadian rhythms links chronobiology to social organization in bees. J. Exp. Biol. 209, 1044-1051 